\title{
EVALUACIÓN DE VIBRACIONES RESONANTES Y CONDICIONES DE SERVICIO DE PUENTES PEATONALES: ESTUDIO DE CASO ACCESO UPTC - TUNJA
}

\author{
A. E. RODRÍGUEZ-SUESCA ${ }^{1,2}$, J. J. CALA-MONROY ${ }^{3}$, \\ J. J. PUENTES-BARON², O. J. GUTIÉRREZ-JUNCO ${ }^{2}$ \\ ${ }^{1}$ Departamento de Mecánica de Estructuras, Universidad de Granada (UGR), Granada, España. \\ ${ }^{2}$ Escuela de Ingeniería Civil, Universidad Pedagógica y Tecnológica de Colombia (UPTC), Tunja, Colombia. \\ ${ }^{3}$ Consultoría y Supervisión de Estructuras, Tunja, Colombia.
}

\begin{abstract}
RESUMEN
A pesar del avance actual de las herramientas que se tienen para diseñar estructuras adecuadas y del sin número de casos documentados del problema de las vibraciones excesivas en estructuras, los puentes peatonales tienden con regularidad a diseñarse para trabajar en frecuencias de vibración cercanos o iguales a la frecuencia de paso de los peatones, trayendo con esto, una serie de inconvenientes durante su uso, que en muchos casos resulta en una falla anticipada por incumplimiento de sus condiciones de servicio, seguridad o durabilidad. Se presenta un estudio de vibraciones y condiciones de servicio realizado en un puente peatonal de arco superior localizado en un acceso universitario de gran afluencia de peatones en Tunja, Colombia. Diferentes pruebas dinámicas sobre el puente ante el paso de peatones con registro directo de aceleración fueron realizadas con el objetivo de caracterizar el problema de resonancia detectado previamente por los usuarios. Se encontró que el puente presenta varias frecuencias fundamentales en el rango de frecuencias del paso de peatones, por lo que esta estructura se encuentra en un diario estado de resonancia. Los niveles de vibración bajo diferentes condiciones de excitación inducida por peatones indicaron excedencia de los límites de confort para varios de los casos de evaluación.
\end{abstract}

Palabras clave: vibraciones resonantes; puente peatonal; condiciones de servicio; carga peatonal

\begin{abstract}
Despite the advanced tools currently available to design suitable structures and the large number of reported cases of the problem of excessive vibration in structures, footbridges tend to be designed with vibration natural frequencies within the step frequency ranges of pedestrian walking, jogging or running. In many cases, a series of inconveniences in the operation stage are evidenced, which can result in an early failure due to non-compliance with the conditions of service, safety or durability. A study of vibrations and service conditions carried out on an arch-type pedestrian bridge located in a university access with a large flow of pedestrians in Tunja, Colombia. Dynamic tests were performed on the footbridge under different pedestrian loading cases with direct acceleration recording. The purpose of the tests was to characterize the resonance problem, previously detected by footbridge users. It was found thatfoot the footbridge has several fundamental frequencies in the footstep frequency range, so this structure is in a recurring state of resonance. Vibration levels under different conditions of pedestrian-induced excitation indicated exceeding comfort limits for several of the evaluation cases. Keywords: resonant vibrations; footbridge; serviceability; pedestrian loading
\end{abstract}




\section{RESUMO}

Apesar das ferramentas avançadas atualmente disponíveis para projetar estruturas adequadas e o grande número de casos relatados do problema de vibração excessiva em estruturas, as pontes pedonais tendem a ser projetadas com freqüências naturais de vibração dentro das faixas de frequência de passos de pedestres. Em muitos casos, evidencia-se uma série de inconvenientes no estágio de operação, o que pode resultar em uma falha prematura por descumprimento das condições de serviço, segurança ou durabilidade. Um estudo de vibrações e condições de serviço realizado em uma ponte pedestre tipo arco localizada em um acesso universitário com um grande fluxo de pedestres em Tunja, na Colômbia. Testes dinâmicos foram realizados na passarela sob diferentes casos de carregamento de pedestres com gravação de aceleração direta. O objetivo dos testes foi caracterizar o problema de ressonância, previamente detectado pelos usuários da passarela. Verificou-se que a passarela possui várias frequências fundamentais na faixa de frequência de passos, de modo que esta estrutura está em um estado recorrente de ressonância. Os níveis de vibração sob diferentes condições de excitação induzida por pedestres indicaram exceder os limites de conforto para vários dos casos de avaliação.

Palavras-chave: vibrações ressonantes; passarela; condições de serviço; carga pedonal

\section{INTRODUCCIÓN}

El diseño de estructuras requiere que el diseñador considere adecuadamente cuatro cualidades fundamentales de su estructura: 1) la resistencia, capacidad de soporte de cargas; 2) relación rigidezmasa, condiciones de funcionamiento adecuado; 3) ductilidad y/o redundancia estructural, capacidad de evitar un colapso súbito; y 4) durabilidad, capacidad de conservar sus propiedades durante la vida de servicio. Sin embargo y a pesar del sin número de herramientas, documentación y normativas que actualmente se disponen para el manejo adecuado y eficiente de dichas variables, se presenta con mucha recurrencia, problemas de diseño estructural, que en varios casos han resultado en hechos desafortunados. Actualmente, se pueden encontrar estructuras que, a los pocos días de ser puestas en funcionamiento, evidencian fallas, causadas por manejo inadecuado de una o varias de las cuatro propiedades mencionadas. Una de las más desatendidas es la relación rigidez-masa, la cual determina las condiciones de funcionamiento y está ligada a condiciones de seguridad y durabilidad. Es el caso de los puentes o pasarelas peatonales, los cuales comúnmente presentan condiciones inconvenientes de funcionamiento, causados por la poca previsión del control de vibraciones en el diseño, que son finalmente detectadas al final del período de construcción o durante el inicio de la puesta en uso. De una revisión preliminar de una serie de puentes peatonales, recientemente construidos en Colombia, se ha detectado que muchos de ellos trabajan en condiciones de resonancia con la frecuencia del caminar humano, lo que puede ocasionar problemas como: molestia y sensación de inseguridad en el peatón, incremento de las solicitaciones en la estructura y por tanto pérdida del nivel de seguridad, daño incremental, fatiga del material, o finalmente el colapso.

Hace no más de tres décadas, cuando se empezaron a presentar problemas de resonancia en estructuras debido al tránsito de personas, se inició el estudio de las vibraciones inducidas por los humanos y la respuesta de las estructuras a estas excitaciones externas. Desde entonces han aparecido una serie de recomendaciones y regulaciones para tratar de controlar la respuesta dinámica de las estructuras ante la acción de paso de peatones, que imponen una excitación periódica en las tres direcciones. A pesar de esto, actualmente no existe un criterio único y por tanto el control de este aspecto es establecido a juicio del diseñador, quien normalmente evita entrar en estudios específicos y se limita a presentar en memorias de cálculo una frecuencia de vibración acomodada de la estructura, y en otros casos se omite este aspecto en el diseño. Frecuentemente, esta desatención o desconocimiento de la frecuencia de 
vibración de la estructura respecto a la de la inducida por los peatones conduce a construir estructuras con problemas de resonancia ante el caminar, trotar o correr de los usuarios.

Poner a trabajar un puente peatonal en resonancia con el paso de peatones es poco aconsejable por la alta probabilidad que se presente excedencia de los límites de servicio y de seguridad, en razón a la alta amplificación de la respuesta dinámica presente en los bajos valores de amortiguamiento de estas estructuras, construidas normalmente en acero o concreto. Adicionalmente, esto conduce a un alto riesgo de excitación vandálica, que ante el paso de una importante cantidad de peatones podría ocasionar el colapso de la estructura.

De cualquier modo, las regulaciones actuales establecen unos límites admisibles de vibración para servicio en términos de aceleración, lo que permite que la estructura trabaje en frecuencias de resonancia con el paso del peatón, siempre que la vibración sea controlada. Estos límites de servicio están definidos para asegurar niveles de confort, pero también para proteger la integridad de la estructura. Así bien, si el puente peatonal se diseña para trabajar en resonancia con el paso de los peatones sobre él, se deberá: realizar análisis numérico que estime los niveles de respuesta dinámica ante las excitaciones por diferentes arreglos y condiciones de paso de peatones; o efectuar una medición de los niveles de vibración posterior a la construcción.

Se presenta el estudio de caso de un puente peatonal para acceso a un área de centros de educación en Tunja, Colombia, en el cual fueron evidenciadas vibraciones excesivas pocos días después de la puesta en uso y reportadas por los transeúntes del puente. Una evaluación experimental de las condiciones de servicio del puente fue adelantada, buscando encontrar el nivel de excedencia frente a la normativa actual.

\section{DESCRIPCIÓN DEL PUENTE PEATONAL ESTUDIADO}

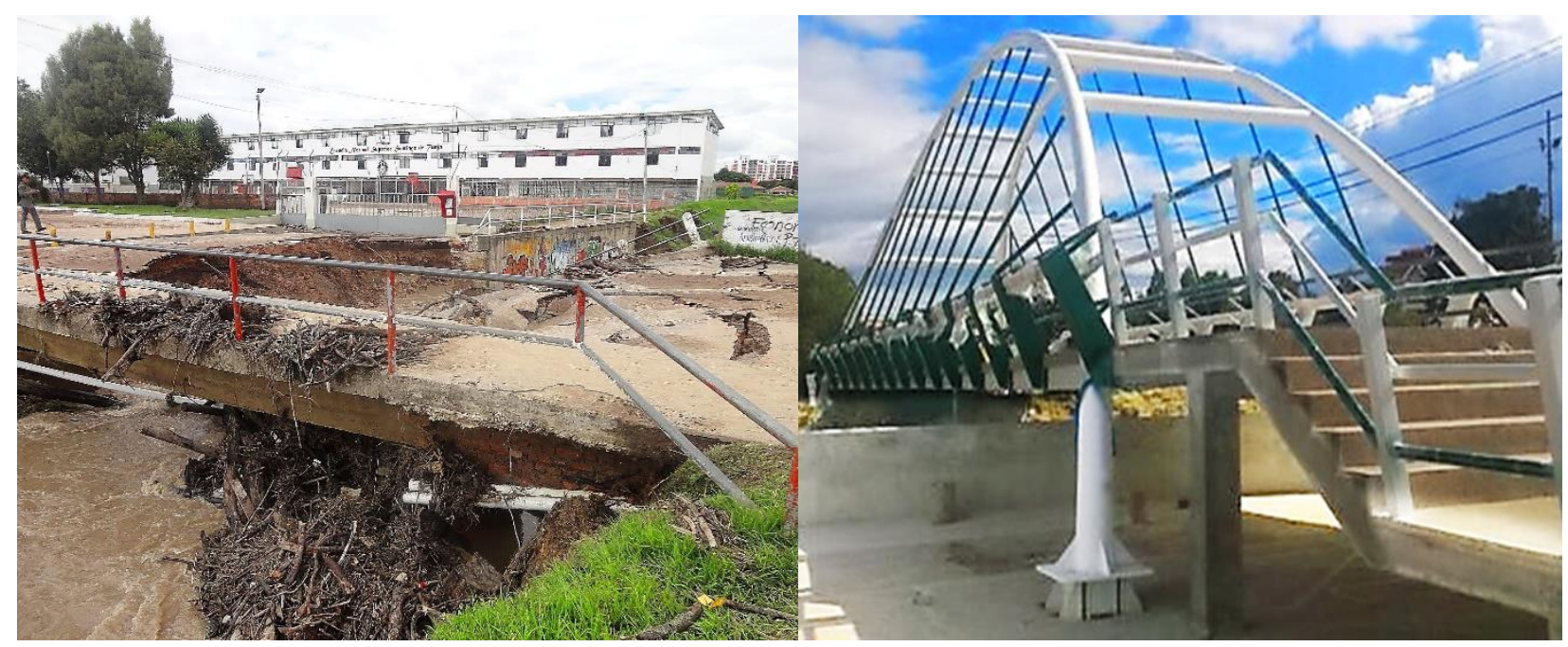

(a)

(b)

Figura 1. a) Colapso puente existente 2012, b) Puente metálico actual

En el 2013, un nuevo puente peatonal sobre el cauce del río La Vega para acceso a un centro universitario y un colegio en la ciudad de Tunja fue construido (acceso principal UPTC), luego del colapso por socavación del puente existente en concreto reforzado durante la fuerte temporada de lluvias ocurrida en el año 2012. En la Figura 1.a se muestra el puente colapsado por la creciente del río La Vega en el 2012, mientras la Figura 1.b muestra el puente metálico por el que fue sustituido. El moderno puente metálico de tablero suspendido de arcos de 30.4 metros de luz fue diseñado y construido con arreglos excéntricos no convencionales en su configuración. Su estructura principal consiste de dos arcos en tubería de acero de sección variable (diámetros de 8" a 12"), los cuales se 
inclinan hacia el eje longitudinal del tablero unos $19^{\circ}$ y se conectan entre si horizontalmente por vigas tubulares de acero. El ancho del tablero del puente es de $6.0 \mathrm{~m}$ en los accesos y de $5.0 \mathrm{~m}$ en el centro de la luz, el cual fue definido en el diseño original de acuerdo al alto flujo de peatones en horas pico del periodo escolar. En la Figura 2 se muestra el esquema general del puente evaluado.
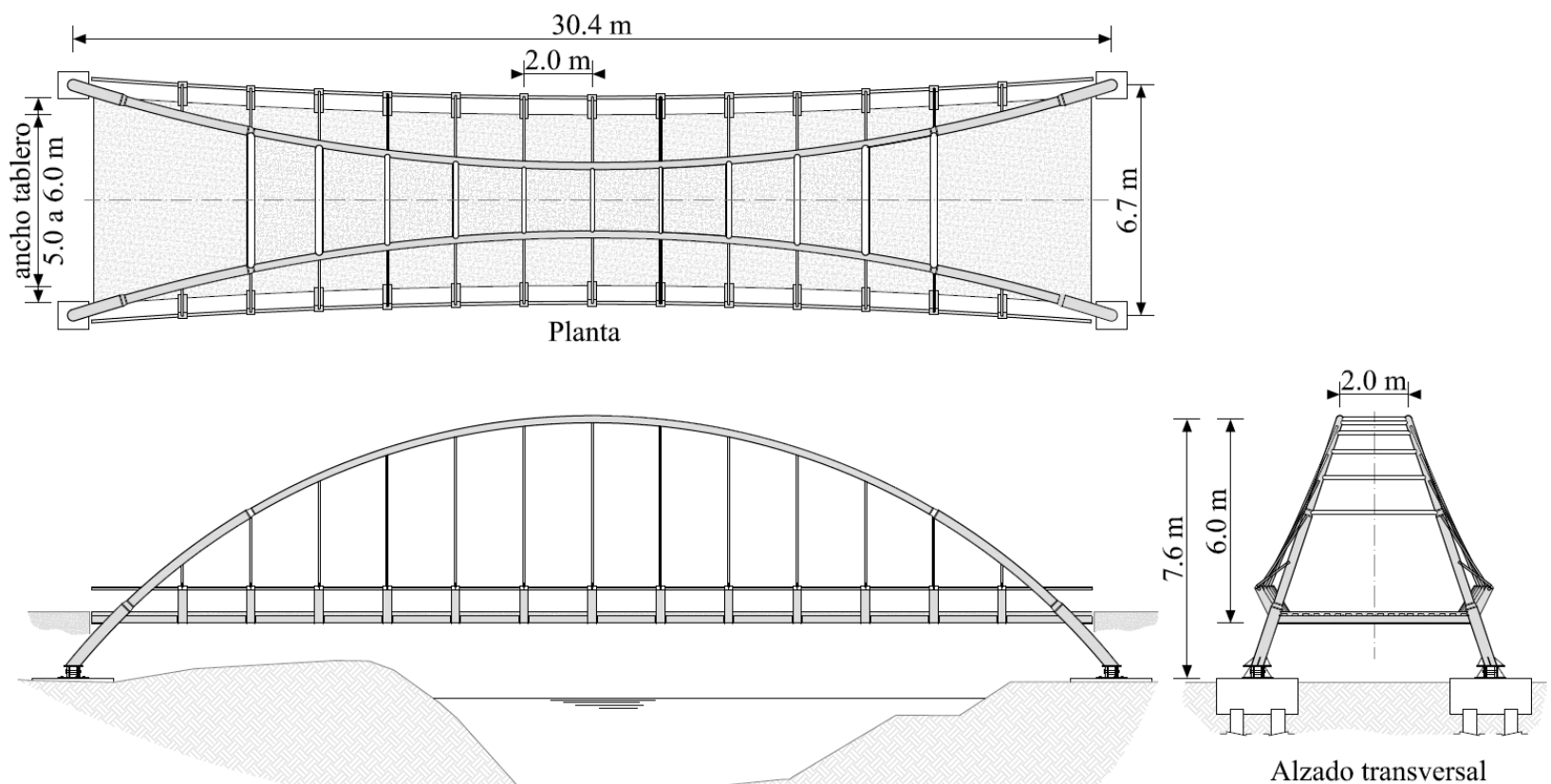

Alzado longitudinal

Figura 2. Geometría general del puente evaluado

\section{IDENTIFICACIÓN MODAL}

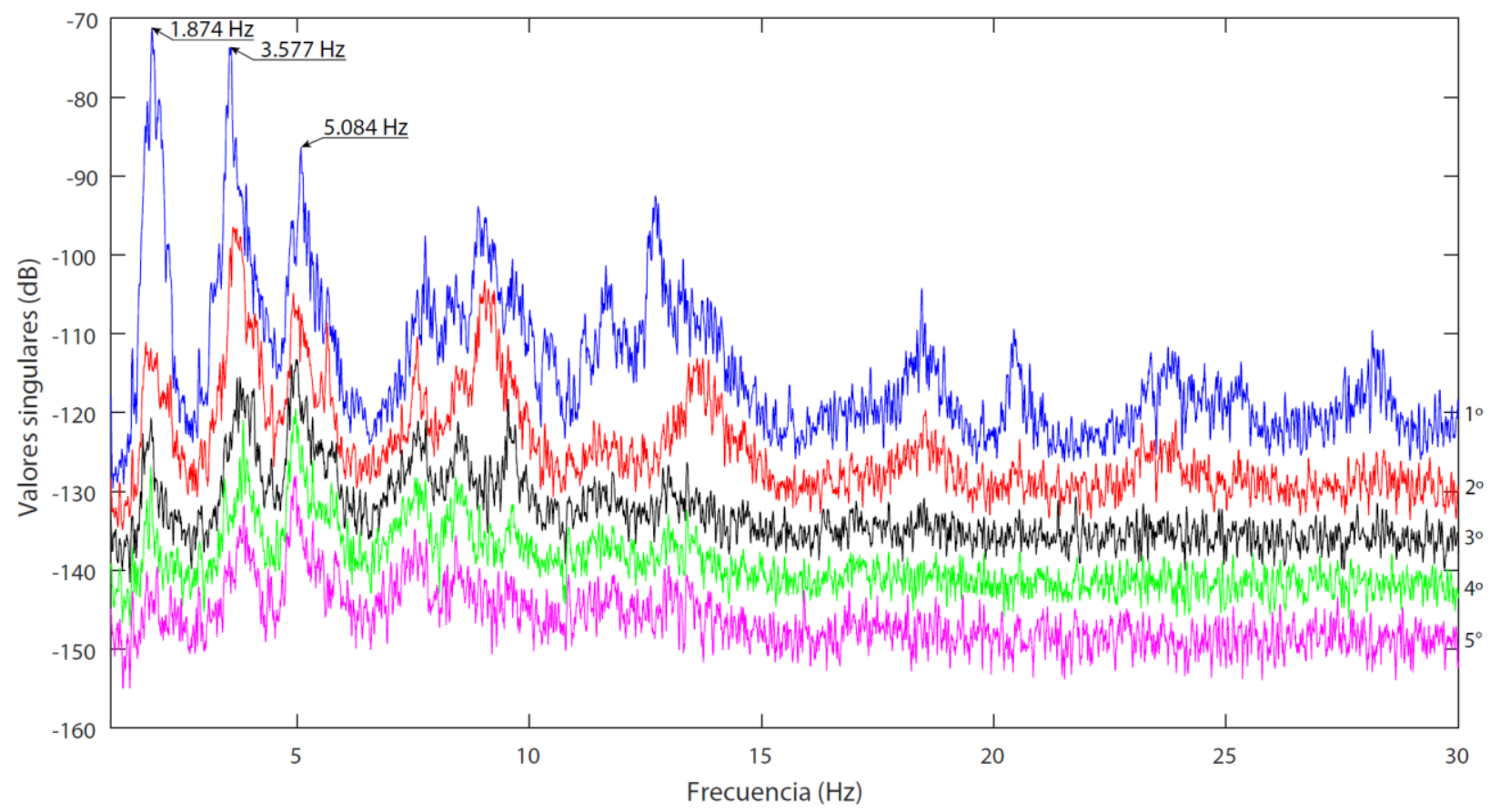

Figura 3. Valores singulares de densidades espectrales (frecuencias de vibración experimentales). 
A fin de evaluar el comportamiento en resonancia percibido por los usuarios del puente, una evaluación experimental de los modos naturales de vibración de la estructura y sus correspondientes frecuencias fueron obtenidos a partir de un análisis modal operacional (OMA - Operational Modal Analysis) (Brincker \& Ventura, 2015). Los parámetros modales fueron extraidos de los registros de vibración ambiental utilizando la metodología de la descomposición del dominio de frecuencia (FDD - Frequency Domain Decomposition) (Brincker, Zhang, \& Andersen, 2000). Estos resultados fueron utilizados para calibrar y mejorar un modelo numérico por computador de la estructura, elaborado para realizar una evaluación analítica de la estructura del puente.

En la Figura 3 se presenta el resultado del análisis experimental efectuado dado en término de los valores singulares de la matriz de densidad espectral evaluada para los registros de aceleración en las tres direcciones en los puntos de medición establecidos. Los picos en la representación de los valores singulares representan las frecuencias predominantes presentes en la estructura del puente. En la Figura 3 se indican tres primeras frecuencias de mayor respuesta ante la excitación ambiental. La frecuencia de $1.874 \mathrm{~Hz}$ representa la frecuencia del primer modo predominante, el cual está asociado con un movimiento longitudinal global de la estructura, pero a su vez, se encontró que se trata de un modo vertical del tablero, como puede observarse en la Figura 4.
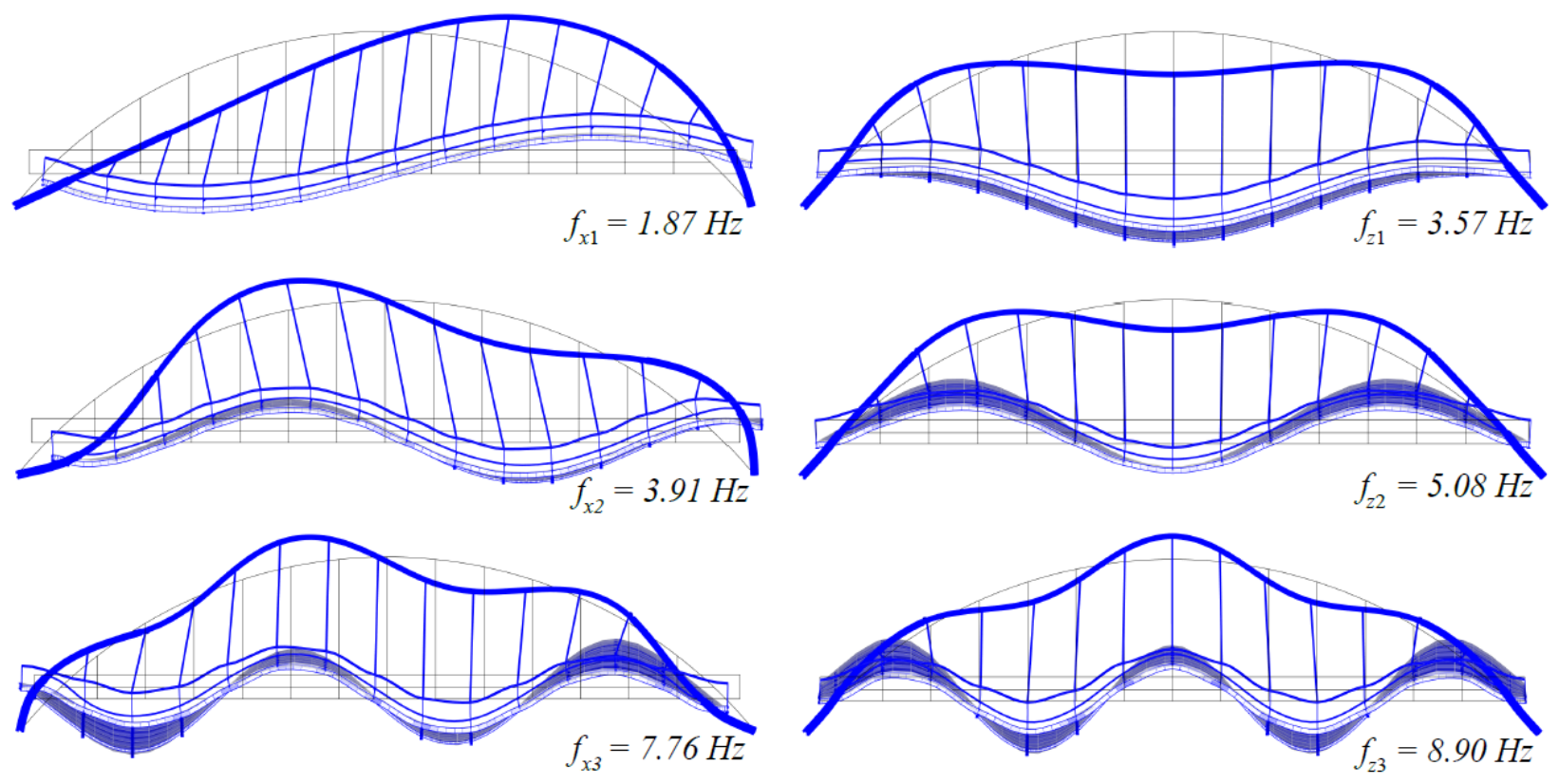

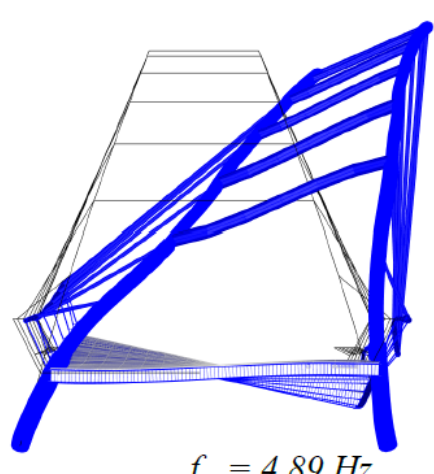

$f_{y 1}=4.89 \mathrm{~Hz}$

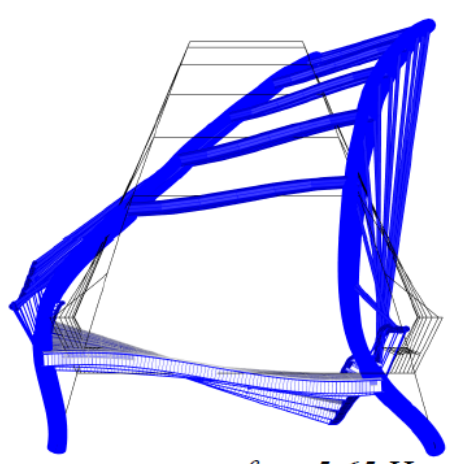

$f_{y 3}=5.65 \mathrm{~Hz}$

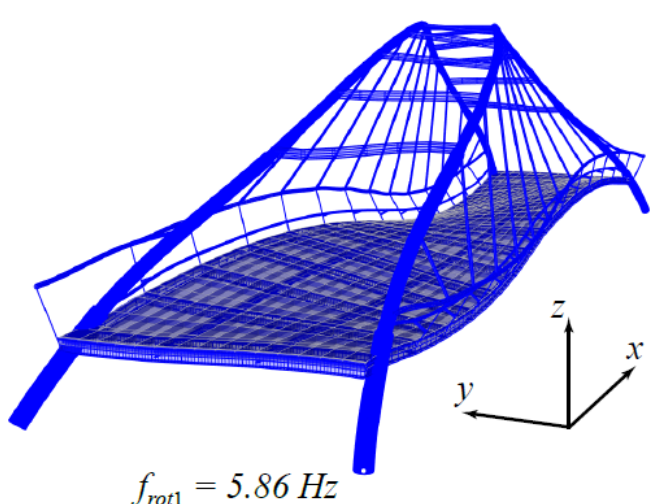

Figura 4. Formas modales y frecuencias de los primeros modos de vibración del puente peatonal

La Figura 4 muestra los primeros modos de vibración del puente peatonal obtenidos con base en el análisis experimental. Estos modos se presentan ordenados de acuerdo a la dirección predominante de 
movimiento, establecida de acuerdo a la participación de masa. Así, se encontró que los modos en la dirección $x$ (longitudinal) presentan un movimiento global predominantemente horizontal, sin embargo, estos modos pueden considerarse también modos verticales del tablero.

\section{EVALUACIÓN DE LA CONDICIÓN DE RESONANCIA}

Se ha encontrado que la frecuencia usual del caminar de los peatones sobre puentes peatonales está entre 1.5 a $2.4 \mathrm{~Hz}$ (Pachi \& Ji, 2005; SYNPEX European research project, 2008). De una medición realizada en el puente peatonal en estudio durante un tiempo de 12 horas en un día típico del periodo de estudio universitario, se encontró un promedio de frecuencia de paso de $1.83 \mathrm{~Hz}$ (110 pasos por minuto). El puente también, eventualmente, está sometido al tránsito de peatones trotando o corriendo con frecuencias entre 2.2 a $3.5 \mathrm{~Hz}$ (contacto discontinuo con el suelo).

En la Tabla 1 se muestra los diferentes rangos de frecuencia natural de vibración de una estructura que son permitidas por las diferentes normativas para evitar que se presente amplificación de la respuesta dinámica por efecto de resonancia con las excitaciones causadas por el tránsito de personas (American Association of State Highway and Transportation Officials - AASHTO, 2009; British Standard Institution - BSI, 2001; Comisión asesora permanente para el régimen de construcciones sismo resistentes, 2010; European Committee for Standardization - CEN, 2005; HIVOSS European research project, 2008; SÉTRA - service d'Études techniques des routes et autoroutes, 2006). Aunque en la normativa actual no existe un consenso en los límites aplicable a puentes peatonales respecto de los rangos de frecuencias adecuadas para funcionamiento relacionadas con el confort y la seguridad, es claro que el paso de peatones impone una excitación periódica que puede incrementar enormemente la respuesta de la edificación y, por tanto, este hecho debe ser de especial atención en el diseño y puesta en funcionamiento de un puente peatonal.

Tabla 1. Frecuencias naturales de vibración permisibles en las estructuras para evitar resonancia

\begin{tabular}{|l|l|l|}
\hline Regulación & Movimiento vertical & Movimiento transversal \\
\hline NSR-10 & $>5 \mathrm{~Hz}$ & $>5 \mathrm{~Hz}$ \\
\hline Eurocódigo 1990 & $>5 \mathrm{~Hz}$ & $>2.5 \mathrm{~Hz}$ \\
\hline SÉTRA & $<1 \mathrm{y}>5 \mathrm{~Hz}$ & $<0.3 \mathrm{y}>2.5 \mathrm{~Hz}$ \\
\hline AASHTO LRFD & $>3 \mathrm{~Hz}$ & $>1.3 \mathrm{~Hz}$ \\
\hline BS 5400 & $<1.5 \mathrm{y}>5 \mathrm{~Hz}$ & $>1.5 \mathrm{~Hz}$ \\
\hline HIVOSS & $<1.25 \mathrm{y}>4.6 \mathrm{~Hz}$ & $<0.5 \mathrm{y}>1.2 \mathrm{~Hz}$ \\
\hline
\end{tabular}

Del análisis de frecuencias realizado se puedo establecer que la estructura del puente peatonal en su primer modo fundamental $\left(f_{x 1}=1.87 \mathrm{~Hz}\right)$ se encuentra trabajando en resonancia con la frecuencia de paso promedio del caminar usual del peatón sobre el puente $(1.83 \mathrm{~Hz})$. Las amplificaciones de la respuesta por resonancia además de presentarse para los casos donde la tasa de paso del peatón es cercana a la frecuencia natural de vibración del puente (1er armónico de la fuerza de paso), es también factible que se amplifique para modos con frecuencias cercanos a dos veces la frecuencia de paso del peatón, donde se dice que la estructura en ese modo responde de forma resonante al segundo armónico de la fuerza del paso. De esta manera, se puede establecer que para los modos de vibración del puente con frecuencias de $3.57 \mathrm{~Hz}$ (primer modo vertical) y $3.91 \mathrm{~Hz}$ (segundo modo longitudinal) el puente presenta amplificación de la respuesta con el segundo armónico de la fuerza de paso de peatones. Se tiene entonces que para los tres primeros modos fundamentales $\left(f_{x 1}=1.87 \mathrm{~Hz}, f_{z 1}=3.57 \mathrm{~Hz}\right.$ y $f_{x 2}=3.91$ $\mathrm{Hz}$ ) la estructura esta fuera del rango de las frecuencias recomendadas por las regulaciones actuales. Es evidente que la estructura del puente estudiado no se rige por el criterio establecido en las normativas actuales para evitar el fenómeno de la resonancia. Por consiguiente, es primordial verificar que el nivel de respuesta de la estructura ante condiciones usuales de tránsito de peatones, no exceda los límites de 
vibración para confort que establecen las normativas, además de verificar la capacidad de la estructura ante las condiciones amplificadas de respuesta dinámica.

\section{NIVEL DE RESPUESTA DINÁMICA ANTE PASO DE PEATONES}

Para evaluar el nivel de confort y seguridad del puente en estudio bajo condiciones reales de servicio, se efectuaron una serie de pruebas dinámicas para diferentes casos de tránsito de peatones que pueden ocurrir durante el funcionamiento normal del puente, como es indicado en guías internacionales SÉTRA y HIVOSS. Las aceleraciones verticales, longitudinales y transversales fueron medidas con sensores de aceleración localizados en el centro y los cuartos de la luz del puente. Tres grupos de pruebas fueron establecidas como se indica en la Tabla 2.

Tabla 2. Respuestas máximas registradas sobre el tablero del puente

\begin{tabular}{|c|c|c|c|c|c|c|c|}
\hline \multirow{2}{*}{ Prueba } & \multirow{2}{*}{ Descripción } & \multirow{2}{*}{ Peatones } & \multirow{2}{*}{$\begin{array}{c}\text { Formación } \\
\text { peatones }\end{array}$} & \multirow{2}{*}{$\begin{array}{c}\text { Frecuencia } \\
\text { de paso }\end{array}$} & \multicolumn{3}{|c|}{\begin{tabular}{|c|} 
Aceleración pico $\left(\mathbf{m} / \mathbf{s}^{2}\right)$ \\
\end{tabular}} \\
\hline & & & & & Longitudinal & Transversal & Vertical \\
\hline \multicolumn{8}{|c|}{ Transito normal } \\
\hline A1 & Vibración ambiental & 0 & - & - & 0.09 & 0.10 & 0.16 \\
\hline $\mathrm{A} 2$ & Flujo peatonal horas no pico & 1 a 5 & aleatorio & no sincrónico & 0.13 & 0.14 & 0.36 \\
\hline A3 & Flujo peatonal horas pico & 1 a 50 & aleatorio & no sincrónico & 0.52 & 0.19 & 0.84 \\
\hline \multicolumn{8}{|c|}{ Transito medio controlado } \\
\hline B1 & Flujo peatonal caminando & 35 & aleatorio & no sincrónico & 0.48 & 0.15 & 0.40 \\
\hline $\mathrm{B} 2$ & Flujo peatonal trotando & 35 & aleatorio & no sincrónico & 1.13 & 0.54 & 2.54 \\
\hline B3 & Flujo peatonal corriendo & 35 & aleatorio & no sincrónico & 1.06 & 0.41 & 4.60 \\
\hline \multicolumn{8}{|c|}{ Frecuencia de paso controlada } \\
\hline $\mathrm{C} 1$ & Caminar sincronizado & 5 & 1 fila transversal & $1.83 \mathrm{~Hz}$ & 0.71 & 0.20 & 1.30 \\
\hline $\mathrm{C} 2$ & Correr & 5 & 1 fila transversal & $\pm 3.40 \mathrm{~Hz}$ & 1.00 & 0.48 & 2.10 \\
\hline $\mathrm{C} 3$ & Caminar sincronizado & 10 & 1 fila transversal & $1.83 \mathrm{~Hz}$ & 1.38 & 0.43 & 2.12 \\
\hline
\end{tabular}

La Tabla 2 lista las aceleraciones máximas registradas sobre el tablero del puente para cada una de las condiciones de carga dinámica por cruce de peatones. Las aceleraciones pico mostradas en la Tabla 2 son las registradas en los puntos de mayor respuesta, que en la mayor parte de los casos resulta en el cuarto de la luz del puente, en coherencia con la forma predominante del primer modo fundamental, $x 1$ (ver Figura 4). Para los casos como el de correr o trotar el primer modo vertical, $z 1$, presento una marcada excitación. El hecho de que el primer modo fundamental sea de carácter longitudinal genera movimientos longitudinales elevados ante las excitaciones verticales producidas por el paso de peatones. Así, aunque las mayores aceleraciones son verticales, en coherencia con la fuerza vertical de excitación, es evidente una elevada respuesta longitudinal que en algunos casos supera el movimiento vertical.

Durante la prueba fue encontrada gran molestia en los peatones, los cuales percibían claramente la resonancia con el caminar, lo que los conducía a intentar acoplarse a la frecuencia predominante del puente, incluso a velocidades diferentes. Si se compara la respuesta del puente de las pruebas de frecuencia de paso sincrónico (pruebas $\mathrm{C}$ ) respecto de la prueba de flujo normal en horas no pico (prueba A2), se encuentra una amplificación de la respuesta un 50\% mayor en el eje longitudinal que en el vertical, lo que indica una clara respuesta en resonancia con el primer modo fundamental $(x 1)$. Este hecho también es evidenciado en las demás pruebas de paso no controlado, en las cuales la respuesta longitudinal se amplifica enormemente en comparación con las demás respuestas. 


\section{EVALUACIÓN DE LAS CONDICIONES DE CONFORT Y SEGURIDAD}

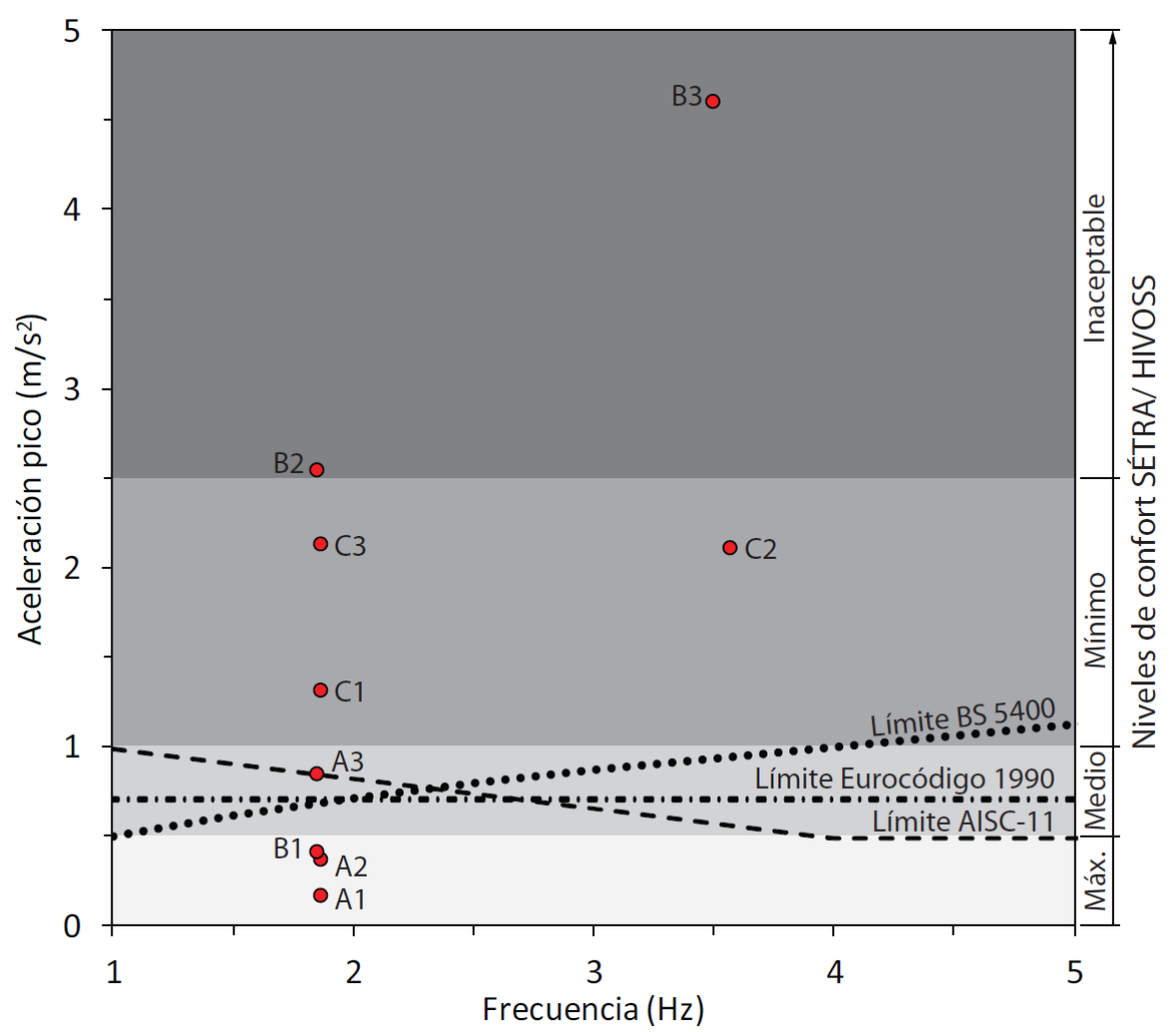

Figura 5. Evaluación de las respuestas verticales máximas registradas en el puente

En las Figuras 5 y 6 se muestra la evaluación de las respuestas máximas del puente (verticales y longitudinales respectivamente) frente a la normativa específica que regula las vibraciones en puentes peatonales (American Institute of Steel Construction Inc., 2003; British Standard Institution - BSI, 2001; European Committee for Standardization - CEN, 2005; HIVOSS European research project, 2008; SÉTRA - service d'Études techniques des routes et autoroutes, 2006). Aunque los criterios de confort para las estructuras son altamente subjetivos y dependen de múltiples factores, en el caso de puentes peatonales, las normativas que contienen esta limitación parecen apuntar a un valor límite máximo vertical de $1.0 \mathrm{~m} / \mathrm{s}^{2}$ (ver límites en Figura 5), para el cual, valores mayores de aceleración de respuesta resultan inadmisibles y, por tanto, se requiere intervención para su reducción. De la evaluación de las pruebas para aceleración vertical (Fig. 5), se encuentra que para el caminar no sincronizado de hasta 50 peatones (pruebas A y B1), las respuestas están justo dentro de los límites máximos normativos $\left(<1.0 \mathrm{~m} / \mathrm{s}^{2}\right)$. Sin embargo, para el transitar sincrónico de 5 o más peatones a una frecuencia de 110 pasos por minuto $(1.83 \mathrm{~Hz})$ se presenta una alta amplificación de la respuesta que lleva a la estructura a respuestas superiores a $1.0 \mathrm{~m} / \mathrm{s}^{2}$. Los casos más críticos se presentan cuando un grupo de 35 personas cruza el puente trotando (B2) o corriendo (B3), donde la respuesta se incrementa enormemente y llegan a superar valores de $2.5 \mathrm{~m} / \mathrm{s}^{2}$, el cual es el límite máximo permitido por las regulaciones SÉTRA y HIVOSS, por lo que estos casos son catalogados como inaceptables. 


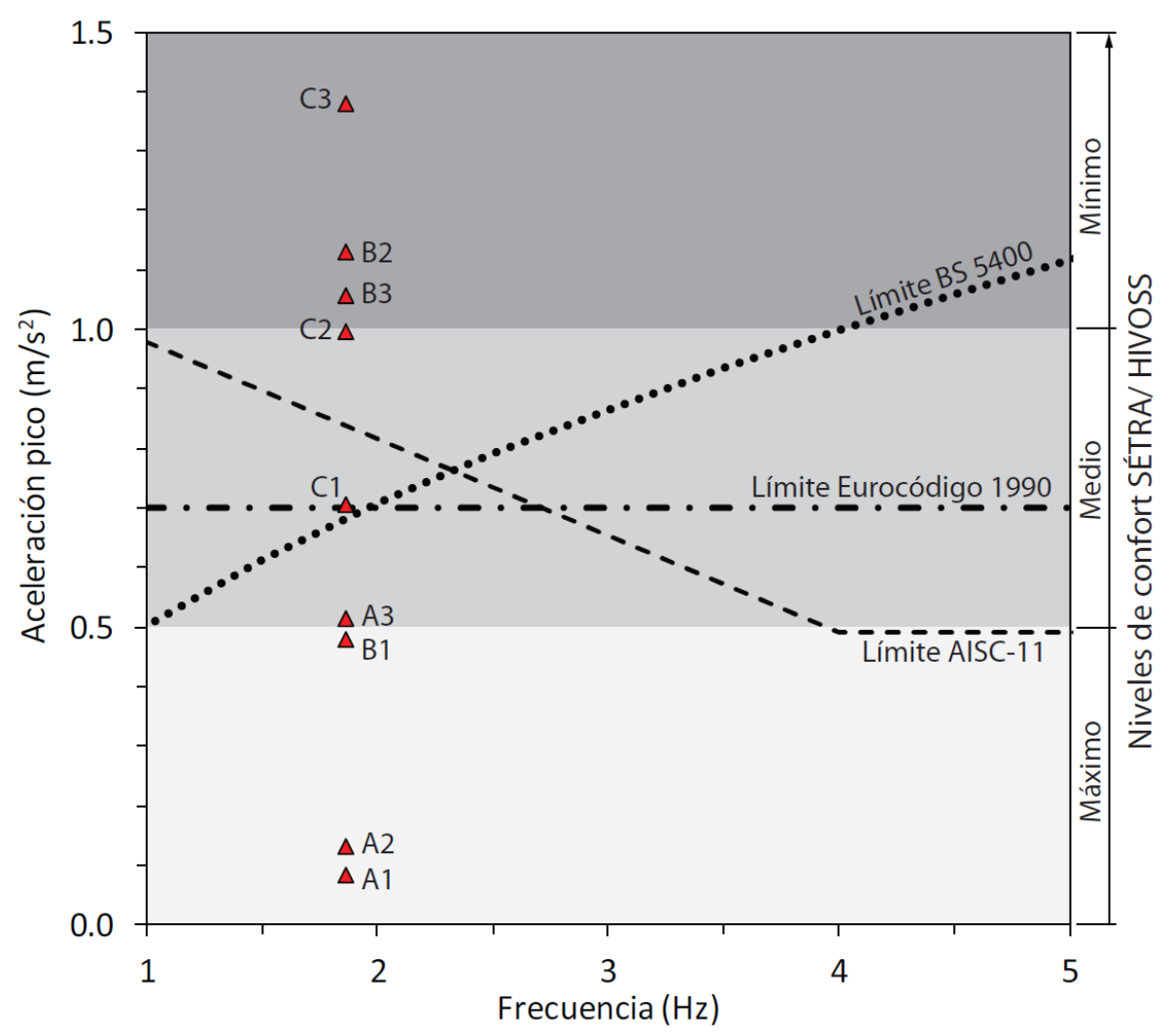

Figura 6. Evaluación de las respuestas longitudinales máximas registradas en el puente

Para el puente en estudio, las aceleraciones horizontales longitudinales son de especial interés dado que están relacionadas con la frecuencia natural del puente en resonancia con el caminar humano (primer modo fundamental). Como se muestra en la Figura 6, las pruebas indican aceleraciones longitudinales por encima de $1.0 \mathrm{~m} / \mathrm{s}^{2}$ para los casos de caminar sincrónico de 10 peatones (C3) y, trotar y correr no sincrónico de 35 peatones (B2 y B3). Para estas condiciones de excedencia se puede establecer un nivel de confort mínimo según las regulaciones SÉTRA y HIVOSS, que indica un nivel de funcionamiento con gran molestia para sus transeúntes. Estas condiciones podrían admitirse siempre que los peatones se localicen longitudinalmente al puente. Sin embargo, dado que el puente es de un ancho considerable existe la posibilidad de evaluar las condiciones de confort para peatones detenidos en el puente y localizados en cualquier dirección, para lo cual los límites establecidos por normativas para el confort por vibraciones horizontales, específicamente para vibraciones laterales a la posición del peatón (European Committee for Standardization - CEN, 2005; HIVOSS European research project, 2008; International Standards Organization - ISO, 2003; SÉTRA - service d'Études techniques des routes et autoroutes, 2006) pueden indicar un incumplimiento de las condiciones de confort para la mayoría de casos evaluados. En la Figura 7 se muestra la comparación de las respuestas máximas longitudinal y transversal frente a los límites establecidos por la normativa para confort lateral del usuario. Dado que las frecuencias transversales del puente están dentro de los rangos permitidos las aceleraciones transversales del puente no representan un problema de confort. Sin embargo, para la mayoría de los casos de tránsito peatonal evaluados, las aceleraciones longitudinales representan un incumplimiento de los niveles de confort horizontal de la normativa existente. 


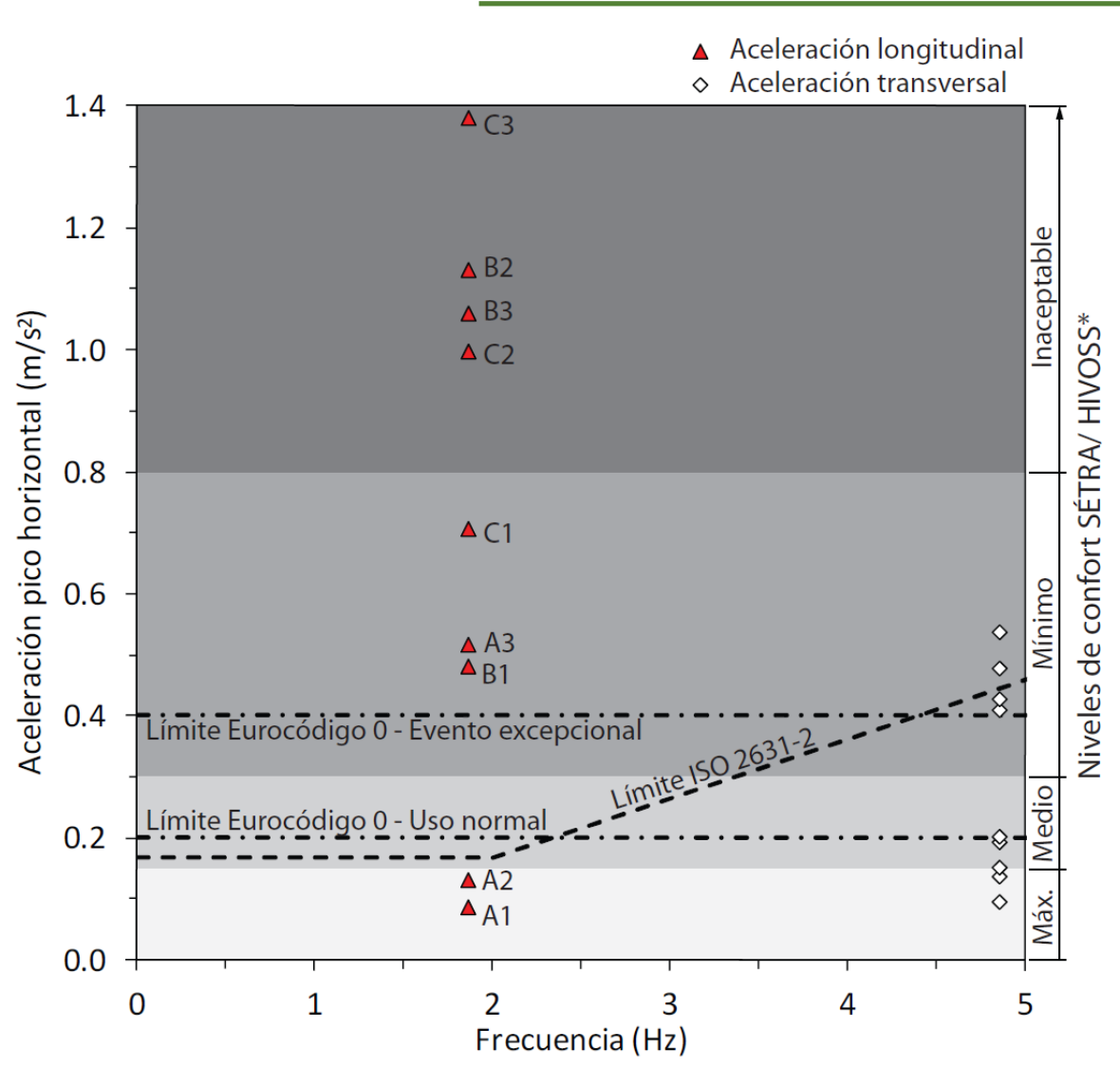

Figura 7. Evaluación de las respuestas horizontales frente a los límites de vibración lateral

\section{CONCLUSIONES}

La investigación desarrollada empleo metodologías experimentales para evaluar las condiciones de servicio de un puente peatonal de alto tráfico peatonal. Se comprobó el estado de resonancia del puente a través de una caracterización de los modos y frecuencias naturales, realizada mediante un análisis modal operacional. Con base en la identificación modal y a través de diferentes pruebas de tránsito peatonal se evaluó el nivel de confort del puente, estableciendo las siguientes conclusiones:

- El puente presenta tres frecuencias menores a $5 \mathrm{~Hz}$ relacionadas con el movimiento vertical y longitudinal, con lo cual el puente esta propenso a sufrir resonancia en diferentes modos ante el primer y segundo armónico del paso usual de peatones.

- El primer modo fundamental de la estructura tiene una frecuencia de $1.87 \mathrm{~Hz}$ que prácticamente coincide con la frecuencia promedio de paso de peatones, 110 pasos por minuto $(1.83 \mathrm{~Hz})$. Debido a esta coincidencia se encontró que el puente trabaja en permanente resonancia ante casi cualquier condición de tránsito peatonal.

- Los niveles de aceleración vertical exceden los límites usuales de confort para condiciones de paso sincrónico previstas por las normativas, mientras las aceleraciones producidas por el tránsito trotando o corriendo de un grupo medio de peatones (35 personas) son inadmisibles, en concordancia con los rangos establecidos por las guías SÉTRA y SEVOSS.

- Las aceleraciones horizontales longitudinales presentan la mayor amplificación por resonancia debido al carácter longitudinal del primer modo fundamental. Para la mayoría de las condiciones de transito probable de peatones, estas aceleraciones longitudinales pueden ser catalogadas como inadmisibles para peatones que estén detenidos sobre el puente. 
- Con base en las condiciones de servicio que exige la normativa actual de puentes peatonales, es imprescindible una intervención de la estructura a fin de cambiar las condiciones desfavorables en las que está funcionando el puente peatonal.

\section{AGRADECIMIENTOS}

La presente investigación fue financiada por la Vicerrectoría de Investigación y Extensión de la Universidad Pedagógica y Tecnológica de Colombia a través de la convocatoria Capital Semilla 2015. Los autores agradecen la participación investigadores y estudiantes vinculados al Grupo de Investigación GICA de la Escuela de Ingeniería Civil de la UPTC quienes colaboraron con el montaje y desarrollo de las pruebas.

\section{REFERENCIAS}

American Association of State Highway and Transportation Officials - AASHTO. (2009). LRFD guide specifications for the design of pedestrian bridges.

American Institute of Steel Construction Inc. (2003). Steel design guide 11: Floor vibrations due to human activity. United States of America.

Brincker, R., \& Ventura, C. (2015). Introduction to operational modal analysis. John Wiley \& Sons.

Brincker, R., Zhang, L., \& Andersen, P. (2000). Modal identification from ambient responses using frequency domain decomposition. In Proceedings of the 18 International Modal Analysis Conference (IMAC) (pp. 625-630). San Antonio, Texas.

British Standard Institution - BSI. (2001). BS 5400-2. Steel, concrete and composite bridges - Part 2: Specification for loads. Appendix C: Vibration serviceability requirements for foot and cycle track bridges. London.

Comisión asesora permanente para el régimen de construcciones sismo resistentes. (2010). NSR-10. Reglamento Colombiano Construcción Sismo Resistente. Ley 400 de 1997, Decreto 33 de 1998, decreto 926 de 2010. Asociación Colombiana de Ingeniería Sísmica.

European Committee for Standardization - CEN. (2005). Eurocode 1990. Basis of structural design. Annex A2: Application for bridges. Eurocode.

HIVOSS European research project. (2008). Human-induced vibration of steel structures: design of footbridges, guideline and background document. Luxembourg.

International Standards Organization - ISO. (2003). ISO 2631-2. Mechanical vibration and shock Evaluation of human exposure to whole-body vibration. Part 2: vibrations in buildings (1 Hz to $80 \mathrm{~Hz}$ ). Pachi, A., \& Ji, T. (2005). Frequency and velocity of people walking. Structural Engineer, 84(3), 3640.

SÉTRA - service d'Études techniques des routes et autoroutes. (2006). Assessment of vibrational behaviour of footbridges under pedestrian loading. Paris - France.

SYNPEX European research project. (2008). Advanced load models for synchronous pedestrian excitation and optimised design guidelines for steel footbridges. 